\title{
Encoding Timed Models as Uniform Labeled Transition Systems *
}

\author{
Marco Bernardo ${ }^{1} \quad$ Luca Tesei $^{2}$ \\ ${ }^{1}$ Dipartimento di Scienze di Base e Fondamenti - Università di Urbino - Italy \\ ${ }^{2}$ Scuola di Scienze e Tecnologie - Università di Camerino - Italy
}

\begin{abstract}
We provide a unifying view of timed models such as timed automata, probabilistic timed automata, and Markov automata. The timed models and their bisimulation semantics are encoded in the framework of uniform labeled transition systems. In this unifying framework, we show that the timed bisimilarities present in the literature can be reobtained and that a new bisimilarity, of which we exhibit the modal logic characterization, can be introduced for timed models including probabilities. We finally highlight similarities and differences among the models.
\end{abstract}

\section{Introduction}

Several extensions of classical automata have been proposed in the last twenty years to model timed aspects of the behavior of systems and to support the verification of hard and soft real-time constraints. The first of these extensions is given by timed automata (TA) [1]. They are equipped with clock variables that measure the passage of time within states, while transitions are instantaneous, may be subject to clock-based guards, and may reset the value of some clocks.

A subsequent extension is that of probabilistic timed automata (PTA) [12]. They are TA where the destination of every transition is a function that associates with each state the probability of being the target state. This allows for the representation both of nondeterministic choices and of probabilistic choices, and enables the investigation of properties such as the probability of executing certain activities within a given deadline is not lower than a given threshold.

The semantics of a TA/PTA can be defined in terms of a variant of labeled transition system (LTS) [11] together with a notion of bisimulation [8]. The characteristic of the underlying variant of LTS is that of having uncountably many states, as any of these states essentially corresponds to a pair composed of a TA/PTA state and a vector of clock values each taken from $\mathbb{R}_{\geq 0}$.

A more recent extension is constituted by Markov automata (MA) [7], in which the probabilistic flavor of PTA transitions is retained, while temporal aspects are described through exponentially distributed random variables rather than deterministic quantities. Since exponential distributions enjoy the memoryless property, an MA no longer needs clocks and hence can be directly viewed as a variant of LTS whose states correspond to the MA states.

* Work partially supported by the MIUR-PRIN Project CINA and the European Commission FP7-ICT-FET Proactive Project TOPDRIM (grant agreement no. 318121). 
In order to emphasize similarities and differences among the various models, in this paper we provide a unifying view of TA, PTA, and MA by encoding all of them as uniform labeled transition systems (ULTRAS) [4]. This is a recently developed framework that has proven to be well suited for uniformly representing different models - ranging from LTS models to discrete-/continuous-time Markov chains and Markov decision processes without/with internal nondeterminism together with their behavioral equivalences.

The paper is organized as follows. In Sect. 2, we recall the notion of ULTRAS and we extend it in order to deal with uncountable state spaces. In Sect. 3, we encode as ULTRAS the variant of LTS underlying TA and we show that the corresponding bisimilarity in $[16,20]$ coincides with a suitable instance of the bisimilarity for ULTRAS. In Sect. 4, we reuse the same encoding to handle TA. In Sect. 5, we encode as ULTRAS the variant of LTS underlying PTA and we show that two different bisimilarities can be defined: the one in [19] and a new one for which we exhibit a modal logic characterization. In Sects. 6 and 7, we reuse almost the same encoding to handle PTA and MA, respectively. Finally, in Sect. 8 we draw some conclusions about the considered timed models.

\section{Revisiting the Definition of ULTRAS}

The definition of ULTRAS given in [4] was based on a set of states and a set of transition-labeling actions that are at most countable. When dealing with TA and PTA models whose time domain is $\mathbb{R}_{\geq 0}$, the underlying LTS models turn out to have uncountably many states and actions. Therefore, we need to extend the definition of ULTRAS by admitting uncountable sets of states and actions, in a way that preserves the results in [4].

Every ULTRAS is parameterized with respect to a set $D$, whose values are interpreted as different degrees of one-step reachability, and a preorder $\sqsubseteq_{D}$ equipped with minimum $\perp_{D}$, which denotes unreachability. In this paper, we consider the set $[S \rightarrow D]_{\mathrm{cs}}$ of countable-support functions from a set $S$ to $D$, i.e., the set of functions $\mathcal{D}: S \rightarrow D$ whose support $\operatorname{supp}(\mathcal{D})=\left\{s \in S \mid \mathcal{D}(s) \neq \perp_{D}\right\}$

is at most countable. As in [4], when $S$ is a set of states, every element $\mathcal{D}$ of $[S \rightarrow D]_{\mathrm{cs}}$ is interpreted as a next-state distribution function and $\operatorname{supp}(\mathcal{D})$ represents the set of reachable states.

Definition 1. Let $\left(D, \sqsubseteq_{D}, \perp_{D}\right)$ be a preordered set equipped with a minimum. $A$ uniform labeled transition system on $\left(D, \sqsubseteq_{D}, \perp_{D}\right)$, or $D$-ULTRAS for short, is a triple $\mathcal{U}=(S, A, \longrightarrow)$ where $S$ is a possibly uncountable set of states, $A$ is a possibly uncountable set of actions, and $\longrightarrow \subseteq S \times A \times[S \rightarrow D]_{\mathrm{cs}}$ is a transition relation. We say that the $D$-ULTRAS $\mathcal{U}$ is functional iff $\longrightarrow$ is a total function from $S \times A$ to $[S \rightarrow D]_{\mathrm{cs}}$.

Every transition $(s, a, \mathcal{D})$ is written $s \stackrel{a}{\longrightarrow} \mathcal{D}$, where $\mathcal{D}\left(s^{\prime}\right)$ is a $D$-value quantifying the degree of reachability of $s^{\prime}$ from $s$ via that transition and $\mathcal{D}\left(s^{\prime}\right)=\perp_{D}$ means that $s^{\prime}$ is not reachable with that transition. If the $D$-ULTRAS is functional, we shall write $\mathcal{D}_{s, a}\left(s^{\prime}\right)$ to denote the same $D$-value. 
A $D$-ULTraS can be depicted as a directed graph-like structure in which vertices represent states and action-labeled edges represent action-labeled transitions. Given a transition $s \stackrel{a}{\longrightarrow} \mathcal{D}$, the corresponding $a$-labeled edge goes from the vertex representing $s$ to a set of vertices linked by a dashed line, each of which represents a state $s^{\prime} \in \operatorname{supp}(\mathcal{D})$ and is labeled with $\mathcal{D}\left(s^{\prime}\right)$. Should $\mathcal{D}\left(s^{\prime}\right)=\perp_{D}$ for all states $s^{\prime}$ - which may happen when the considered D-ULTRAS is functional the transition would not be depicted at all. A $\mathbb{B}$-ULTRAS is shown on the righthand side of Fig. 1, where $\mathbb{B}=\{\perp, \top\}$ is the support set of the Boolean algebra, $\perp$ (false) denotes unreachability, $T$ (true) denotes reachability, and $\perp \sqsubseteq_{\mathbb{B}} T$.

In [4], various equivalences were defined over ULTRAS and shown to coincide in most cases with those appeared in the literature of nondeterministic, probabilistic, stochastic, and mixed models. Since in this paper we focus on bisimilarity, we shall recall only the definition of bisimilarity for ULTRAS. This definition, like the one of the other equivalences, is parameterized with respect to a measure function that expresses the degree of multi-step reachability of a set of states in terms of values taken from a preordered set equipped with minimum. In the following, we call trace an element $\alpha$ of $A^{*}$ and we denote by $\varepsilon$ the empty trace, by "|_|" the operation that computes the length of a trace, and by "_o _" the operation that concatenates two traces.

Definition 2. Let $\mathcal{U}=(S, A, \longrightarrow)$ be a $D$-ULTraS, $n \in \mathbb{N}, s_{i} \in S$ for $0 \leq i \leq n$, and $a_{i} \in A$ for $1 \leq i \leq n$. We say that $s_{0} \stackrel{a_{1}}{\longrightarrow} s_{1} \stackrel{a_{2}}{\longrightarrow} s_{2} \ldots s_{n-1} \stackrel{a_{n}}{\longrightarrow} s_{n}$ is a computation of $\mathcal{U}$ of length $n$ that goes from $s_{0}$ to $s_{n}$ and is labeled with trace $a_{1} a_{2} \ldots a_{n}$ iff for all $i=1, \ldots, n$ there exists a transition $s_{i-1} \stackrel{a_{i}}{\longrightarrow} \mathcal{D}_{i}$ such that $s_{i} \in \operatorname{supp}\left(\mathcal{D}_{i}\right)$.

Definition 3. Let $\mathcal{U}=(S, A, \longrightarrow)$ be a D-ULTraS and $\left(M, \sqsubseteq_{M}, \perp_{M}\right)$ be a preordered set equipped with a minimum. A measure function on $\left(M, \sqsubseteq_{M}, \perp_{M}\right)$ for $\mathcal{U}$, or $M$-measure function for $\mathcal{U}$, is a function $\mathcal{M}_{M}: S \times A^{*} \times 2^{S} \rightarrow M$ such that the value of $\mathcal{M}_{M}\left(s, \alpha, S^{\prime}\right)$ is defined by induction on $|\alpha|$ and depends only on the reachability of a state in $S^{\prime}$ from state $s$ through computations labeled with trace $\alpha$.

Definition 4. Let $\mathcal{U}=(S, A, \longrightarrow)$ be a $D$-ULTraS and $\mathcal{M}_{M}$ be an $M$-measure function for $\mathcal{U}$. An equivalence relation $\mathcal{B}$ over $S$ is an $\mathcal{M}_{M}$-bisimulation iff, whenever $\left(s_{1}, s_{2}\right) \in \mathcal{B}$, then for all actions $a \in A$ and groups of equivalence classes $\mathcal{G} \in 2^{S / \mathcal{B}}$ it holds that:

$$
\mathcal{M}_{M}\left(s_{1}, a, \bigcup \mathcal{G}\right)=\mathcal{M}_{M}\left(s_{2}, a, \bigcup \mathcal{G}\right)
$$

where $\bigcup \mathcal{G}$ is the union of all the equivalence classes in $\mathcal{G}$. We say that $s_{1}, s_{2} \in S$ are $\mathcal{M}_{M}$-bisimilar, written $s_{1} \sim_{\mathrm{B}, \mathcal{M}_{M}} s_{2}$, iff there exists an $\mathcal{M}_{M}$-bisimulation $\mathcal{B}$ over $S$ such that $\left(s_{1}, s_{2}\right) \in \mathcal{B}$.

The preordered structure $\left(M, \sqsubseteq_{M}, \perp_{M}\right)$ for multi-step reachability used in the definition of the equivalence does not necessarily coincide with the preordered structure $\left(D, \sqsubseteq_{D}, \perp_{D}\right)$ for one-step reachability used in the definition of the model. In [4], various cases were illustrated that demonstrate the necessity of keeping the two structures separate to retrieve certain equivalences. 
The definition of bisimilarity is given in the style of [14], i.e., it requires a bisimulation to be an equivalence relation. However, it deals with arbitrary groups of equivalence classes rather than only with individual equivalence classes. As shown in [4], working with groups of equivalence classes provides an adequate support to models in which nondeterminism and quantitative aspects coexist. In particular, it gives rise to new probabilistic bisimulation equivalences that have interesting logical characterizations (see the references in [4]).

\section{Encoding Timed LTS Models}

Timed processes can be represented as models enriched with timing information. Following the orthogonal-time approach ${ }^{1}$ of [16], we consider an extension of LTS called timed labeled transition system (TLTS). In this model, functional aspects (i.e., process activities assumed to be instantaneous) are separate from temporal aspects (i.e., time passing) by means of two distinct transition relations: one labeled with actions and the other labeled with amounts of time. Since we are interested in TLTS models obtained from TA, we shall consider $\mathbb{R}_{\geq 0}$ as time domain and allow for uncountably many states and actions.

Definition 5. A timed labeled transition system (TLTS) is a quadruple ( $S, A$, $\longrightarrow, \leadsto)$ where $S$ is a possibly uncountable set of states, $A$ is a possibly uncountable set of actions, and:

$-\longrightarrow \subseteq S \times A \times S$ is an action-transition relation such that for all $s \in S$ and $a \in \bar{A}$ it holds that $\left\{s^{\prime} \in S \mid\left(s, a, s^{\prime}\right) \in \longrightarrow\right\}$ is at most countable.

$-\leadsto \subseteq S \times \mathbb{R}_{>0} \times S$ is a time-transition relation satisfying $(s, 0, s) \in \leadsto$ [0-delay], $\left(s, t, s_{1}^{\prime}\right) \in \leadsto \wedge\left(s, t, s_{2}^{\prime}\right) \in \leadsto \Longrightarrow s_{1}^{\prime}=s_{2}^{\prime}$ [time determinism], and $\left(s, t_{1}, s^{\prime}\right) \in \leadsto \wedge\left(s^{\prime}, t_{2}, s^{\prime \prime}\right) \in \leadsto \Longrightarrow\left(s, t_{1}+t_{2}, s^{\prime \prime}\right) \in \leadsto[$ time additivity $]$.

Every action-transition $\left(s, a, s^{\prime}\right)$ is written $s \stackrel{a}{\longrightarrow} s^{\prime}$ and means that $s$ can reach $s^{\prime}$ by executing action $a$, whilst every time-transition $\left(s, t, s^{\prime}\right)$ is written $s \stackrel{t}{\sim} s^{\prime}$ and means that $s$ can evolve into $s^{\prime}$ after an amount of time equal to $t$.

Following [20], we can merge the two transition relations into a single one by adding a special time-elapsing action $\epsilon(t)$ for every $t \in \mathbb{R}_{\geq 0}$. With this in mind, it is immediate to see that a TLTS can be encoded as a functional B-ULTRAS.

Definition 6. Let $(S, A, \longrightarrow, \leadsto)$ be a TLTS. Its corresponding functional $\mathbb{B}$-ULTraS $\mathcal{U}=\left(S, A_{\mathcal{U}}, \longrightarrow \mathcal{U}\right)$ is defined by letting:

- $A_{\mathcal{U}}=A \cup\left\{\epsilon(t) \mid t \in \mathbb{R}_{\geq 0}\right\}$.

$-s \stackrel{a}{\longrightarrow} \mathcal{U} \mathcal{D}_{s, a}$ for all $s \in S$ and $a \in A_{\mathcal{U}}$.

$-\mathcal{D}_{s, a}\left(s^{\prime}\right)= \begin{cases}\top & \text { if } a \in A \text { and } s \stackrel{a}{\longrightarrow} s^{\prime}, \text { or } a=\epsilon(t) \text { and } \stackrel{t}{\sim} s^{\prime} \\ \perp & \text { otherwise }\end{cases}$ for all $s^{\prime} \in S$.

\footnotetext{
${ }^{1}$ As opposed to the integrated-time approach, in which process activities are assumed
} to be durational: see $[6,3]$ for an overview of both approaches in different settings. 


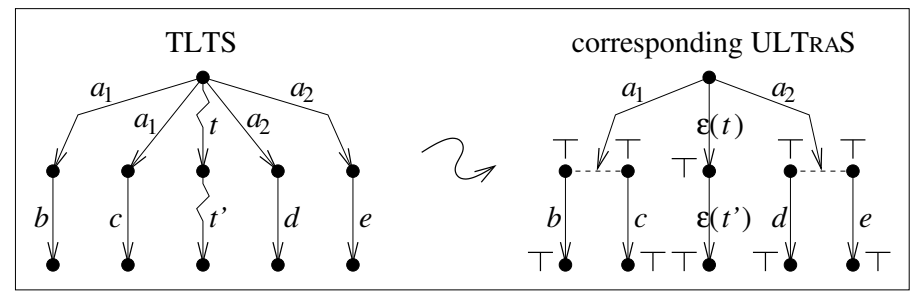

Fig. 1. Translation of a TLTS exhibiting both external and internal nondeterminism

If a TLTS state has a certain number of differently labeled outgoing actiontransitions, then those transitions are retained in the corresponding functional $\mathbb{B}$-ULTRAS. In other words, external nondeterminism in the original model is preserved by the resulting model. In contrast, internal nondeterminism is encoded within the target countable-support functions of the transitions of the resulting model. Indeed, if a TLTS state has several identically labeled outgoing action-transitions, then a single transition is generated in the corresponding functional $\mathbb{B}$-ULTRAS, in which several states are assigned $T$ as reachability value. The encoding of both forms of nondeterminism is exemplified in Fig. 1.

A notion of bisimilarity for timed processes was introduced in [16,20], where the congruence property and an equational characterization were also studied. The decidability of timed bisimilarity was established in [5].

Definition 7. Let $(S, A, \longrightarrow, \sim)$ be a TLTS. A relation $\mathcal{B}$ over $S$ is a timed bisimulation iff, whenever $\left(s_{1}, s_{2}\right) \in \mathcal{B}$, then for all actions $a \in A$ and amounts of time $t \in \mathbb{R}_{\geq 0}$ it holds that:

- For each $s_{1} \stackrel{a}{\longrightarrow} s_{1}^{\prime}$ (resp. $\left.s_{2} \stackrel{a}{\longrightarrow} s_{2}^{\prime}\right)$ there exists $s_{2} \stackrel{a}{\longrightarrow} s_{2}^{\prime}$ (resp. $s_{1} \stackrel{a}{\longrightarrow} s_{1}^{\prime}$ ) such that $\left(s_{1}^{\prime}, s_{2}^{\prime}\right) \in \mathcal{B}$.

- For each $s_{1} \stackrel{t}{\sim} s_{1}^{\prime}$ (resp. $s_{2} \stackrel{t}{\sim} s_{2}^{\prime}$ ) there exists $s_{2} \stackrel{t}{\sim} s_{2}^{\prime}$ (resp. $s_{1} \stackrel{t}{\sim} s_{1}^{\prime}$ ) such that $\left(s_{1}^{\prime}, s_{2}^{\prime}\right) \in \mathcal{B}$.

We say that $s_{1}, s_{2} \in S$ are timed bisimilar, written $s_{1} \sim_{\mathrm{TB}} s_{2}$, iff there exists a timed bisimulation $\mathcal{B}$ over $S$ such that $\left(s_{1}, s_{2}\right) \in \mathcal{B}$.

Timed bisimilarity over TLTS models is captured by $\sim_{\mathbb{B}, \mathcal{M}_{\mathbb{B}}}$ over the corresponding functional $\mathbb{B}$-ULTRAS models, where measure function $\mathcal{M}_{\mathbb{B}}$ is defined in Table 1. When $\alpha=a \circ \alpha^{\prime}$, the measure function considers each possible next state $s^{\prime}$ by examining whether it is reachable from $s$ via $a\left(\mathcal{D}_{s, a}\left(s^{\prime}\right)\right)$ and it can reach a state in $S^{\prime}$ via $\alpha^{\prime}\left(\mathcal{M}_{\mathbb{B}}\left(s^{\prime}, \alpha^{\prime}, S^{\prime}\right)\right)$. If this is the case for at least one of the possible next states $s^{\prime}$, then $\mathcal{M}_{\mathbb{B}}\left(s, \alpha, S^{\prime}\right)=\top$, otherwise $\mathcal{M}_{\mathbb{B}}\left(s, \alpha, S^{\prime}\right)=\perp$. Note that, for TLTS models, the preordered structure of the corresponding ULTRAS models coincides with the preordered structure of the measure function.

Theorem 1. Let $(S, A, \longrightarrow, \sim)$ be a TLTS and $\mathcal{U}=\left(S, A_{\mathcal{U}}, \longrightarrow \mathcal{U}\right)$ be the corresponding functional $\mathbb{B}$-ULTRAS. For all $s_{1}, s_{2} \in S$ :

$$
s_{1} \sim_{\mathrm{TB}} s_{2} \Longleftrightarrow s_{1} \sim_{\mathrm{B}, \mathcal{M}_{\mathbb{B}}} s_{2}
$$




$$
\mathcal{M}_{\mathbb{B}}\left(s, \alpha, S^{\prime}\right)=\left\{\begin{array}{ll|}
\bigvee_{s^{\prime} \in S} \mathcal{D}_{s, a}\left(s^{\prime}\right) \wedge \mathcal{M}_{\mathbb{B}}\left(s^{\prime}, \alpha^{\prime}, S^{\prime}\right) & \text { if } \alpha=a \circ \alpha^{\prime} \\
\top & \text { if } \alpha=\varepsilon \text { and } s \in S^{\prime} \\
\perp & \text { if } \alpha=\varepsilon \text { and } s \notin S^{\prime}
\end{array}\right.
$$

Table 1. Measure function for functional B-ULTRAS models representing TLTS models

\section{Encoding Timed Automata}

Timed automata (TA) [1] extend classical automata by introducing clock variables, or simply clocks, that measure the passage of time. They all advance at the same speed and take values in $\mathbb{R}_{\geq 0}$. A clock valuation $\nu \in \mathcal{V}_{\mathcal{X}}$ over a finite set of clocks $\mathcal{X}$ is a total function from $\mathcal{X}$ to $\mathbb{R}_{\geq 0}$. Given a valuation $\nu$ and a delay $t \in \mathbb{R}_{\geq 0}$, we let $\nu+t$ denote the valuation mapping each clock $x \in \mathcal{X}$ into $\nu(x)+t$. A reset $\gamma$ is a set of clocks in $\mathcal{X}$ whose value is set back to zero. For a valuation $\nu$ and a reset $\gamma$, we let $\nu \backslash \gamma(x)=0$ if $x \in \gamma$ and $\nu \backslash \gamma(x)=\nu(x)$ if $x \notin \gamma$.

In TA, time elapses in states, called locations, as long as invariant conditions associated with the locations themselves hold. These are constraints on the values of the clocks through which notions such as urgency or laziness of actions can be expressed [9]. In contrast, the execution of an action transition is considered instantaneous. Transitions are guarded, i.e., enabled/disabled, by constraints on the values of the clocks, and can reset the value of some clocks.

The set $\Psi_{\mathcal{X}}$ of clock constraints over a finite set of clocks $\mathcal{X}$ is defined by the following grammar: $\psi::=x \# c \mid \psi \wedge \psi$ where $x \in \mathcal{X}, c \in \mathbb{N}$, and $\# \in\{<\rangle,, \leq$, $\geq,=\}$. Clock constraints are assessed over clock valuations. The satisfaction of a clock constraint $\psi$ by a valuation $\nu$, denoted by $\nu \models \psi$, is defined as follows: (i) $\nu \models x \# c$ iff $\nu(x) \# c$, (ii) $\nu \models \psi_{1} \wedge \psi_{2}$ iff $\nu \models \psi_{1}$ and $\nu \models \psi_{2}$. The given syntax for constraints is minimal; the so-called diagonal clock constraints of the form $x-y \# c$ can be simulated by using more locations and the constraints of the given form [2]. An invariant condition is a clock constraint with the property of being past-closed, i.e., for all valuations $\nu$ and delays $t \in \mathbb{R}_{\geq 0}$ it holds that $\nu+t \models \psi \Longrightarrow \nu \models \psi$.

Definition 8. A timed automaton (TA) is a tuple $(L, A, \mathcal{X}, I, \longrightarrow)$ where $L$ is a finite set of locations, $A$ is a set of actions, $\mathcal{X}$ is a finite set of clocks, $I$ is a function mapping each location into an invariant condition, and $\longrightarrow \subseteq$ $L \times \Psi_{\mathcal{X}} \times A \times 2^{\mathcal{X}} \times L$ is a transition relation .

Every transition is written $\ell \stackrel{\psi, a, \gamma}{\longrightarrow} \ell^{\prime}$ where $\ell$ is the source location, $\psi$ is the guard, $a$ is the action label, $\gamma$ is the clock reset, and $\ell^{\prime}$ is the target location.

The semantics of a TA is given in terms of a TLTS. Thus, it is natural to encode a TA as a functional $\mathbb{B}$-ULTRAS generated by using the same conditions defining the TA semantics.

Definition 9. Let $(L, A, \mathcal{X}, I, \longrightarrow)$ be a TA. Its corresponding functional $\mathbb{B}$-ULTRAS $\mathcal{U}=\left(S, A_{\mathcal{U}}, \longrightarrow \mathcal{U}\right)$ is defined by letting: 


$$
\begin{aligned}
& -S=\left\{(\ell, \nu) \in L \times \mathcal{V}_{\mathcal{X}}|\nu|=I(\ell)\right\} . \\
& -A_{\mathcal{U}}=A \cup\left\{\epsilon(t) \mid t \in \mathbb{R}_{\geq 0}\right\} . \\
& -(\ell, \nu) \stackrel{a}{\longrightarrow} \mathcal{U} \mathcal{D}_{(\ell, \nu), a} \text { for all }(\ell, \nu) \in S \text { and } a \in A \mathcal{U} . \\
& -\mathcal{D}_{(\ell, \nu), a}\left(\ell^{\prime}, \nu^{\prime}\right)= \begin{cases}\top & \text { if } a \in A, \ell \stackrel{\psi, a, \gamma}{\longrightarrow} \ell^{\prime}, \nu \models \psi, \nu^{\prime}=\nu \backslash \gamma, \nu^{\prime}=I\left(\ell^{\prime}\right) \\
\top & \text { if } a=\epsilon(t), \ell^{\prime}=\ell, \nu^{\prime}=\nu+t, \nu^{\prime} \models I\left(\ell^{\prime}\right) \\
\perp & \text { otherwise }\end{cases} \\
& \quad \text { for all }\left(\ell^{\prime}, \nu^{\prime}\right) \in S .
\end{aligned}
$$

Timed bisimilarity over TA models is defined in terms of the underlying TLTS models. Therefore, we can reuse both Def. 7 and Table 1, so that Thm. 1 also applies to functional $\mathbb{B}$-ULTRAS models corresponding to TA models.

\section{Encoding Probabilistic Timed LTS Models}

A probabilistic extension of the TLTS model (PTLTS) was introduced in [19]. Following the simple probabilistic automaton model of [18], the action-transition relation is transformed into a probabilistic action-transition relation. This means that a PTLTS action transition, instead of leading to a single target state, has a probability distribution over target states assigning each such state the probability of being reached. Therefore, the choice among several outgoing action transitions from the same state is nondeterministic, whereas the choice of the target state for the selected transition is probabilistic. Given a possibly uncountable set $S$, we denote by $\operatorname{Distr}_{\mathrm{cs}}(S)$ the set of probability distributions $\mathcal{D}$ over $S$ whose support $\operatorname{supp}(\mathcal{D})=\{s \in S \mid \mathcal{D}(s)>0\}$ is at most countable.

While in [19] there is a single transition relation and hence each transition is also labeled with the duration of the corresponding action, here we stick to the orthogonal-time approach and hence keep using two transition relations: a probabilistic one labeled with actions and a deterministic one labeled with amounts of time. We prefer to do so for two reasons. Firstly, this allows us to use a consistent notation and model structure throughout the paper. Secondly, separating functional aspects from time aspects simplifies the development of weak behavioral equivalences, as has been shown in the deterministic time case $[20,17,13]$ and in the stochastic time case $[10,7]$.

Definition 10. $A$ probabilistic timed labeled transition system (PTLTS) is a quadruple $(S, A, \longrightarrow, \sim)$ where $S$ is a possibly uncountable set of states, $A$ is a possibly uncountable set of actions, and:

- $\longrightarrow \subseteq S \times A \times \operatorname{Distr}_{\mathrm{cs}}(S)$ is a probabilistic action-transition relation.

$-\leadsto \subseteq S \times \mathbb{R}_{\geq 0} \times S$ is a time-transition relation satisfying 0 -delay, time determinism, and time additivity.

Every action-transition $(s, a, \mathcal{D})$ is written $s \stackrel{a}{\longrightarrow} \mathcal{D}$ - which is already in the ULTRAS transition format - whilst every time-transition $\left(s, t, s^{\prime}\right)$ is written $s \stackrel{t}{\sim} s^{\prime}$. As in the TLTS case, we can merge the two transition relations into a single one by adding a special time-elapsing action $\epsilon(t)$ for every $t \in \mathbb{R}_{\geq 0}$, such that 


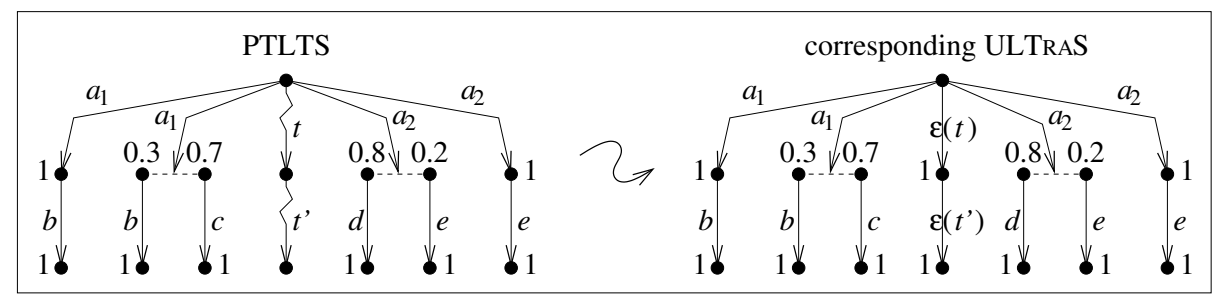

Fig. 2. Translation of a PTLTS exhibiting both external and internal nondeterminism

the target distributions of the transitions labeled with such actions concentrate all the probability mass into a single state. At this point, it is straightforward to encode a PTLTS as an $\mathbb{R}_{[0,1]}$-ULTRAS, which relies on the usual ordering for real numbers - with 0 denoting unreachability - and is not necessarily functional due to the coexistence of probability and internal nondeterminism [4]. In the following, given $s \in S$ we denote by $\delta_{s}$ the Dirac distribution for $s$, where $\delta_{s}(s)=1$ and $\delta_{s}\left(s^{\prime}\right)=0$ for all $s^{\prime} \in S \backslash\{s\}$.

Definition 11. Let $(S, A, \longrightarrow, \leadsto)$ be a PTLTS. Its corresponding $\mathbb{R}_{[0,1]}$-ULTRAS $\mathcal{U}=\left(S, A_{\mathcal{U}}, \longrightarrow \mathcal{U}\right)$ is defined by letting:

- $A_{\mathcal{U}}=A \cup\left\{\epsilon(t) \mid t \in \mathbb{R}_{\geq 0}\right\}$.

$-s \stackrel{a}{\longrightarrow} \mathcal{U} \mathcal{D}$ for each $s \stackrel{a}{\longrightarrow} \mathcal{D}$.

$-s \stackrel{\epsilon(t)}{\longrightarrow} \mathcal{U} \delta_{s^{\prime}}$ for each $\stackrel{t}{\sim} s^{\prime}$.

Different from the TLTS encoding, both external nondeterminism and internal nondeterminism in the original PTLTS are preserved in the corresponding $\mathbb{R}_{[0,1]}$-ULTraS. This is exemplified in Fig. 2.

A notion of bisimilarity for probabilistic timed processes was introduced in [19], where a modal logic characterization and a decision procedure were also studied. Below, we reformulate the definition in the orthogonal-time framework and we let $\mathcal{D}(C)=\sum_{s \in C} \mathcal{D}(s)$ for $\mathcal{D} \in \operatorname{Distr}_{\mathrm{cs}}(S)$ and $C \subseteq S$.

Definition 12. Let $(S, A, \longrightarrow, \leadsto)$ be a PTLTS. An equivalence relation $\mathcal{B}$ over $S$ is a probabilistic timed bisimulation iff, whenever $\left(s_{1}, s_{2}\right) \in \mathcal{B}$, then for all actions $a \in A$ and amounts of time $t \in \mathbb{R}_{\geq 0}$ it holds that:

- For each $s_{1} \stackrel{a}{\longrightarrow} \mathcal{D}_{1}$ there exists $s_{2} \stackrel{a}{\longrightarrow} \mathcal{D}_{2}$ such that for all equivalence classes $C \in S / \mathcal{B}$ it holds that $\mathcal{D}_{1}(C)=\mathcal{D}_{2}(C)$.

- For each $s_{1} \stackrel{t}{\sim} s_{1}^{\prime}$ there exists $s_{2} \stackrel{t}{\sim} s_{2}^{\prime}$ such that $\left(s_{1}^{\prime}, s_{2}^{\prime}\right) \in \mathcal{B}$.

We say that $s_{1}, s_{2} \in S$ are probabilistic timed bisimilar, written $s_{1} \sim_{\mathrm{PTB}} s_{2}$, iff there exists a probabilistic timed bisimulation $\mathcal{B}$ over $S$ such that $\left(s_{1}, s_{2}\right) \in \mathcal{B}$.

It is relatively easy to see that the relation $\sim_{\mathrm{PTB}}$ coincides with the following bisimulation equivalence defined over $\mathbb{R}_{[0,1]}$-ULTRAS models corresponding to 


$\mathcal{M}_{2 \cdot\left[\mathbb{R}_{[0,1]}\right.}\left(s, \alpha, S^{\prime}\right)= \begin{cases}\bigcup_{a}\left\{\sum_{s^{\prime} \in S} \mathcal{D}\left(s^{\prime}\right) \cdot p_{s^{\prime}}\right. & \left.p_{s^{\prime}} \in \mathcal{M}_{2 \cdot \mathbb{R}_{[0,1]}}\left(s^{\prime}, \alpha^{\prime}, S^{\prime}\right)\right\} \\ \{1\} & \text { if } \alpha=a \circ \alpha^{\prime} \text { and there exists } s \stackrel{a}{\longrightarrow} \mathcal{D} \\ \{0\} & \text { if } \alpha=\varepsilon \text { and } s \in S^{\prime} \\ & \text { if } \alpha=a \circ \alpha^{\prime} \text { and there is no } s \stackrel{a}{\longrightarrow} \mathcal{D} \\ & \text { or } \alpha=\varepsilon \text { and } s \notin S^{\prime}\end{cases}$

Table 2. Measure function for $\mathbb{R}_{[0,1]}$-ULTRAS models representing PTLTS models

PTLTS models. The equivalence below is called group-distribution bisimilarity because it compares entire distributions of reaching groups of equivalence classes. Given two related states, for each transition of one of the two states there must exist an equally labeled transition of the other state such that, for every group of equivalence classes, the two transitions have the same probability of reaching a state in that group. In other words, the two transitions must be fully matching, i.e., they must match with respect to all groups.

Definition 13. Let $\mathcal{U}=\left(S, A_{\mathcal{U}}, \longrightarrow \mathcal{U}\right)$ be the $\mathbb{R}_{[0,1]}$-ULTRAS corresponding to a $\operatorname{PTLTS}(S, A, \longrightarrow, \sim)$. An equivalence relation $\mathcal{B}$ over $S$ is a probabilistic timed group-distribution bisimulation iff, whenever $\left(s_{1}, s_{2}\right) \in \mathcal{B}$, then for all actions $a \in A_{\mathcal{U}}$ it holds that:

- For each $s_{1} \stackrel{a}{\longrightarrow} \mathcal{U} \mathcal{D}_{1}$ there exists $s_{2} \stackrel{a}{\longrightarrow} \mathcal{U} \mathcal{D}_{2}$ such that for all groups of equivalence classes $\mathcal{G} \in 2^{S / \mathcal{B}}$ it holds that $\mathcal{D}_{1}(\bigcup \mathcal{G})=\mathcal{D}_{2}(\bigcup \mathcal{G})$.

We say that $s_{1}, s_{2} \in S$ are probabilistic timed group-distribution bisimilar, written $s_{1} \sim \sim_{\mathrm{PTB}, \mathrm{dis}} s_{2}$, iff there exists a probabilistic timed group-distribution bisimulation $\mathcal{B}$ over $S$ such that $\left(s_{1}, s_{2}\right) \in \mathcal{B}$.

Theorem 2. Let $(S, A, \longrightarrow, \leadsto)$ be a PTLTS and $\mathcal{U}=\left(S, A_{\mathcal{U}}, \longrightarrow \mathcal{U}\right)$ be the corresponding $\mathbb{R}_{[0,1]}$-ULTRAS. For all $s_{1}, s_{2} \in S$ :

$$
s_{1} \sim_{\mathrm{PTB}} s_{2} \Longleftrightarrow s_{1} \sim_{\mathrm{PTB}, \mathrm{dis}} s_{2}
$$

The relation $\sim_{\text {PTB }}$ over PTLTS models has been expressed as $\sim_{\text {PTB,dis }}$ in the ULTRAS setting, but cannot be captured by any instantiation of the general bisimilarity for ULTRAS given in Def. 4. In the case of probabilistic timed processes, a natural measure function is the one defined in Table 2. Denoting by $2^{\mathbb{R}} \cdot[0,1]$ the set of nonempty subsets of $\mathbb{R}_{[0,1]}$, this measure function associates a suitable element of $2 \mathbb{R}^{\mathbb{R}}[0,1]$ with every triple composed of a source state $s$, a trace $\alpha$, and a set of destination states $S^{\prime}$. The set $\mathcal{M}_{2^{\mathbb{R}}[0,1]}\left(s, \alpha, S^{\prime}\right)$ contains for each possible way of resolving nondeterminism the probability of performing a computation that is labeled with trace $\alpha$ and leads to a state in $S^{\prime}$ from state $s$. It is worth pointing out that, while the considered ULTRAS models are based on the preordered structure $\left(\mathbb{R}_{[0,1]}, \leq, 0\right)$, the measure function relies on the different preordered structure $\left(2{ }^{\mathbb{R}}[0,1], \sqsubseteq,\{0\}\right)$ where $R_{1} \sqsubseteq R_{2}$ means $\inf R_{1} \leq \inf R_{2}$ and $\left|R_{1}\right| \leq\left|R_{2}\right|$ (the latter condition ensures $\{0\}$ being the minimum). 


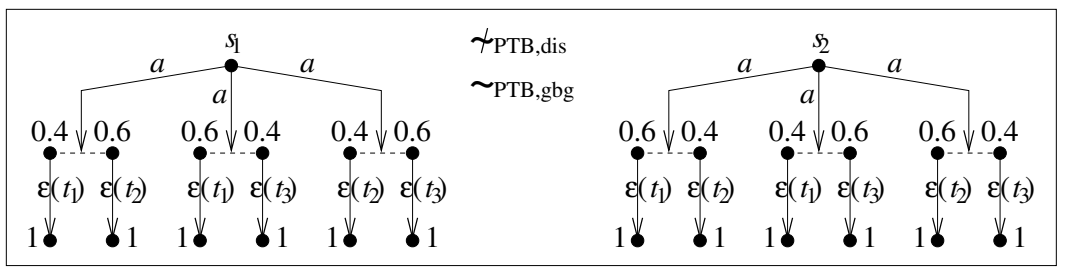

Fig. 3. Counterexample showing that $\sim_{\mathrm{PTB}}$,gbg is strictly coarser than $\sim_{\mathrm{PTB}}$ dis

The resulting bisimilarity $\sim_{\mathrm{B}, \mathcal{M}_{2} \mathbb{R}_{[0,1]}}$ captures the following equivalence that we call group-by-group bisimilarity because it considers a single group of equivalence classes at a time. Technically speaking, this amounts to anticipating the quantification over groups (underlined in Def. 13) with respect to the quantification over transitions. In this way, a transition departing from one of two related states is allowed to be matched, with respect to the probabilities of reaching different groups, by several different transitions departing from the other state. In other words, partially matching transitions are allowed.

Definition 14. Let $\mathcal{U}=\left(S, A_{\mathcal{U}}, \longrightarrow \mathcal{U}\right)$ be the $\mathbb{R}_{[0,1]}$-ULTrAS corresponding to a PTLTS $(S, A, \longrightarrow, \leadsto)$. An equivalence relation $\mathcal{B}$ over $S$ is a probabilistic timed group-by-group bisimulation iff, whenever $\left(s_{1}, s_{2}\right) \in \mathcal{B}$, then for all actions $a \in A_{\mathcal{U}}$ and for all groups of equivalence classes $\mathcal{G} \in 2^{S / \mathcal{B}}$ it holds that:

- For each $s_{1} \stackrel{a}{\longrightarrow} \mathcal{U} \mathcal{D}_{1}$ there exists $s_{2} \stackrel{a}{\longrightarrow} \mathcal{U} \mathcal{D}_{2}$ such that $\mathcal{D}_{1}(\bigcup \mathcal{G})=\mathcal{D}_{2}(\bigcup \mathcal{G})$.

We say that $s_{1}, s_{2} \in S$ are probabilistic timed group-by-group bisimilar, written $s_{1} \sim_{\mathrm{PTB}, \mathrm{gbg}} s_{2}$, iff there exists a probabilistic timed group-by-group bisimulation $\mathcal{B}$ over $S$ such that $\left(s_{1}, s_{2}\right) \in \mathcal{B}$.

Theorem 3. Let $\mathcal{U}=\left(S, A_{\mathcal{U}}, \longrightarrow \mathcal{U}\right)$ be the $\mathbb{R}_{[0,1]}$-ULTRAS corresponding to a $\operatorname{PTLTS}(S, A, \longrightarrow, \leadsto)$. For all $s_{1}, s_{2} \in S$ :

$$
s_{1} \sim_{\text {PTB }, \text { gbg }} s_{2} \Longleftrightarrow s_{1} \sim_{\mathrm{B}, \mathcal{M}_{2 .}[0,1]} s_{2}
$$

In presence of internal nondeterminism, $\sim_{\mathrm{PTB}, \mathrm{gbg}}$ strictly contains $\sim_{\mathrm{PTB}, \mathrm{dis}}$, as shown in Fig. 3. Indicating states with the actions they enable, it holds that $s_{1} \chi_{\mathrm{PTB}, \mathrm{dis}} s_{2}$ because the group distribution of the leftmost $a$-transition of $s_{1}$ - which assigns probability 1 to each group containing both the $\epsilon\left(t_{1}\right)$ state and the $\epsilon\left(t_{2}\right)$-state, probability 0.4 to each group containing the $\epsilon\left(t_{1}\right)$-state but not the $\epsilon\left(t_{2}\right)$-state, probability 0.6 to each group containing the $\epsilon\left(t_{2}\right)$-state but not the $\epsilon\left(t_{1}\right)$-state, and probability 0 to any other group - is not matched by the group distribution of any of the three $a$-transitions of $s_{2}$. In contrast, $s_{1} \sim$ PTB,gbg $s_{2}$. For instance, the leftmost $a$-transition of $s_{1}$ is matched by the leftmost $a$-transition of $s_{2}$ with respect to every group containing both the $\epsilon\left(t_{1}\right)$ state and the $\epsilon\left(t_{2}\right)$-state, the central $a$-transition of $s_{2}$ with respect to every group containing the $\epsilon\left(t_{1}\right)$-state but not the $\epsilon\left(t_{2}\right)$-state, and the rightmost $a$ transition of $s_{2}$ with respect to every group containing the $\epsilon\left(t_{2}\right)$-state but not the $\epsilon\left(t_{1}\right)$-state. 
Theorem 4. Let $\mathcal{U}=\left(S, A_{\mathcal{U}}, \longrightarrow \mathcal{U}\right)$ be the $\mathbb{R}_{[0,1]}$-ULTRAS corresponding to a $\operatorname{PTLTS}(S, A, \longrightarrow, \leadsto)$. For all $s_{1}, s_{2} \in S$ :

$$
s_{1} \sim_{\mathrm{PTB}, \operatorname{dis}} s_{2} \Longrightarrow s_{1} \sim_{\mathrm{PTB}, \mathrm{gbg}} s_{2}
$$

We conclude by exhibiting a modal logic characterization of $\sim_{\mathrm{PTB} \text {,gbg }}$ (and hence of $\sim_{\mathrm{B}, \mathcal{M}_{2} \mathbb{R}_{[0,1]}}$ ). Unlike the characterization of $\sim_{\mathrm{PTB}}\left(\right.$ i.e., $\left.\sim_{\mathrm{PTB}, \mathrm{dis}}\right)$ provided in [19], which relies on an expressive probabilistic extension of HML [8] interpreted over state distributions, here it is sufficient to consider the intervalbased variant IPML of the probabilistic modal logic in [14] with the following syntax: $\phi::=$ true $|\neg \phi| \phi \wedge \phi \mid\langle a\rangle_{\left[p_{1}, p_{2}\right]} \phi$ where $a \in A_{\mathcal{U}}$ and $p_{1}, p_{2} \in \mathbb{R}_{[0,1]}$ such that $p_{1} \leq p_{2}$. A state $s \in S$ belongs to the set $\mathcal{M} \llbracket\langle a\rangle_{\left[p_{1}, p_{2}\right]} \phi \rrbracket$ of states satisfying $\langle a\rangle_{\left[p_{1}, p_{2}\right]} \phi$ iff there exists $s \stackrel{a}{\longrightarrow} \mathcal{U} \mathcal{D}$ such that $p_{1} \leq \mathcal{D}(\mathcal{M} \llbracket \phi \rrbracket) \leq p_{2}$.

Theorem 5. Let $\mathcal{U}=\left(S, A_{\mathcal{U}}, \longrightarrow \mathcal{U}\right)$ be the $\mathbb{R}_{[0,1]}$-ULTRAS corresponding to $a \operatorname{PTLTS}(S, A, \longrightarrow, \sim)$. For all $s_{1}, s_{2} \in S$ it holds that $s_{1} \sim_{\mathrm{PTB}, \mathrm{gbg}} s_{2}$ iff $s_{1}$ and $s_{2}$ satisfy the same formulae of IPML.

\section{Encoding Probabilistic Timed Automata}

Probabilistic timed automata (PTA) [12] extend TA with probabilities. While the passage of time remains deterministic, the target of each action transition becomes a probability distribution. The approach is exactly the one described in Sect. 5 for moving from TLTS models to PTLTS models.

Definition 15. A probabilistic timed automaton (PTA) is a tuple ( $L, A, \mathcal{X}, I$, $\longrightarrow$ ) where $L$ is a finite set of locations, $A$ is a set of actions, $\mathcal{X}$ is a finite set of clocks, $I$ is a function mapping each location into an invariant condition, and $\longrightarrow \subseteq L \times \Psi_{\mathcal{X}} \times A \times \operatorname{Distr}_{\mathrm{cs}}\left(2^{\mathcal{X}} \times L\right)$ is a transition relation .

Every transition is written $\ell \stackrel{\psi, a}{\longrightarrow} \mathcal{D}$ where $\mathcal{D}$ is the probability distribution assigning each pair $\left(\gamma, \ell^{\prime}\right)$ the probability of being reached via that transition.

Like for TA, the semantics of a PTA is given in terms of a PTLTS. Following the same approach used in Sect. 4 , we thus encode a PTA as an $\mathbb{R}_{[0,1]^{-}}$ULTrAS generated by using the same conditions defining the PTA semantics.

Definition 16. Let $(L, A, \mathcal{X}, I, \longrightarrow)$ be a PTA. Its corresponding $\mathbb{R}_{[0,1]}$-ULTRAS $\mathcal{U}=\left(S, A_{\mathcal{U}}, \longrightarrow \mathcal{U}\right)$ is defined by letting:

- $S=\left\{(\ell, \nu) \in L \times \mathcal{V}_{\mathcal{X}}|\nu|=I(\ell)\right\}$.

- $A_{\mathcal{U}}=A \cup\left\{\epsilon(t) \mid t \in \mathbb{R}_{\geq 0}\right\}$.

- $(\ell, \nu) \stackrel{a}{\longrightarrow} \mathcal{U} \mathcal{D}$ for each $\ell \stackrel{\psi, a}{\longrightarrow} \mathcal{D}^{\prime}$ such that $\nu \models \psi$, where for all $\left(\ell^{\prime}, \nu^{\prime}\right) \in S$ $\mathcal{D}\left(\ell^{\prime}, \nu^{\prime}\right)=\sum_{\gamma \in \operatorname{reset}\left(\nu, \nu^{\prime}\right)} \mathcal{D}^{\prime}\left(\gamma, \ell^{\prime}\right)$ with $\operatorname{reset}\left(\nu, \nu^{\prime}\right)=\left\{\gamma \in 2^{\mathcal{X}} \mid \nu \backslash \gamma=\nu^{\prime}\right\}$.

- $(\ell, \nu) \stackrel{\epsilon(t)}{\longrightarrow} \mathcal{U} \delta_{\left(\ell^{\prime}, \nu^{\prime}\right)}$ for $\ell^{\prime}=\ell, \nu^{\prime}=\nu+t, \nu^{\prime} \models I\left(\ell^{\prime}\right)$.

Similar to TA models, probabilistic timed bisimilarity over PTA models is defined in terms of the underlying PTLTS models. Therefore, we can reuse Defs. 12,13 , and 14 as well as Table 2, so that Thms. 2, 3, 4, and 5 also apply to $\mathbb{R}_{[0,1]}$-ULTRAS corresponding to PTA models. 


\section{Encoding Markov Automata}

So far, we have considered timed models in which temporal aspects are described as fixed amounts of time. In other words, in these models the passage of time is represented deterministically. However, in many situations there are fluctuations in the time that elapses between instantaneous activities. When these fluctuations are quantifiable, the passage of time can be represented stochastically.

Due to the simplicity of their mathematical treatment, exponentially distributed random variables are mostly used for a stochastic representation of time. Given one such variable $X$ with parameter $\lambda \in \mathbb{R}_{>0}$, the probability that a duration sampled from $X$ is at most $t \in \mathbb{R}_{\geq 0}$ is given by $\operatorname{Pr}\{X \leq t\}=1-\mathrm{e}^{-\lambda \cdot t}$. The parameter $\lambda$ is said the rate of $X$; its reciprocal is the expected value of $X$.

If several alternative exponentially distributed delays can elapse from a state, the race policy is adopted; the delay that elapses is the one sampling the least duration. It can be shown that the following property $\mathcal{R} \mathcal{P}$ holds in that state: the sojourn time is exponentially distributed with rate given by the sum of the rates of the various delays, with the probability of selecting each such delay being proportional to its rate.

The recently introduced model of Markov automata (MA) [7] can be viewed as a variant of PTA models in which time passing is described through exponentially distributed random variables. An important property of any of these variables is that it enjoys the memoryless property; even if we know that a certain amount of time has already elapsed, the residual time is still quantified by the same exponentially distributed random variable. As a consequence, in this setting there is no need for clocks and hence Markov automata can actually be viewed as a variant of PTLTS models, with exponentially distributed delays (uniquely identified by their rates) in place of deterministic delays.

Definition 17. A Markov automaton (MA) is a quadruple $(S, A, \longrightarrow, \sim)$ where $S$ is a possibly uncountable set of states, $A$ is a possibly uncountable set of actions, and:

$-\longrightarrow \subseteq S \times A \times \operatorname{Distr}_{\mathrm{cs}}(S)$ is a probabilistic action-transition relation .

$-\leadsto \subseteq S \times \mathbb{R}_{>0} \times S$ is a bounded time-transition relation, i.e., for all $s \in S$ it holds that $\left\{s^{\prime} \in S \mid \exists \lambda \in \mathbb{R}_{>0} \cdot\left(s, \lambda, s^{\prime}\right) \in \leadsto\right\}$ is at most countable and $\sum_{\left(s, \lambda, s^{\prime}\right) \in \sim} \lambda<\infty$.

Similar to the PTLTS case, every action-transition $(s, a, \mathcal{D})$ is written $s \stackrel{a}{\longrightarrow} \mathcal{D}$, every time-transition $\left(s, \lambda, s^{\prime}\right)$ is written $s \stackrel{\lambda}{\sim} s^{\prime}$, and we can merge the two transition relations into a single one by adding a special time-elapsing action $\epsilon(\lambda)$ for every $\lambda \in \mathbb{R}_{>0}$. Following the transformation sketched in [7], it is straightforward to encode an MA as a not necessarily functional $\mathbb{R}_{[0,1]}$-ULTRAS, in which the race policy is represented based on $\mathcal{R} \mathcal{P}$. For each state having outgoing timetransitions, we generate a single time-elapsing transition - instead of one such transition for each original delay - such that its rate $\lambda$ is the sum of the rates identifying the original delays and its target distribution assigns to every state a probability proportional to the rate at which that state can be reached. 


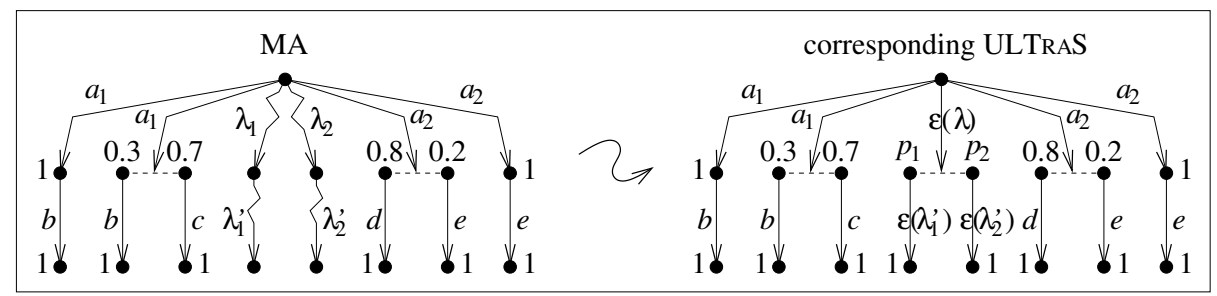

Fig. 4. Translation of an MA $\left(\lambda=\lambda_{1}+\lambda_{2}, p_{1}=\lambda_{1} / \lambda, p_{2}=\lambda_{2} / \lambda\right)$

Definition 18. Let $(S, A, \longrightarrow, \sim)$ be an $M A$. Its corresponding $\mathbb{R}_{[0,1]}$-ULTRAS $\mathcal{U}=\left(S, A_{\mathcal{U}}, \longrightarrow \mathcal{U}\right)$ is defined by letting:

- $A_{\mathcal{U}}=A \cup\left\{\epsilon(\lambda) \mid \lambda \in \mathbb{R}_{>0}\right\}$.

$-s \stackrel{a}{\longrightarrow} \mathcal{U} \mathcal{D}$ for each $s \stackrel{a}{\longrightarrow} \mathcal{D}$.

$-s \stackrel{\epsilon(\lambda)}{\longrightarrow} \mathcal{U} \mathcal{D}$ for all $s \in S$ having outgoing time-transitions, where $\lambda=\sum_{s \lambda^{\lambda^{\prime}} s^{\prime}} \lambda^{\prime}$ and $\mathcal{D}\left(s^{\prime}\right)=\sum_{s \underset{s}{\lambda^{\prime}} s^{\prime}} \lambda^{\prime} / \lambda$ for all $s^{\prime} \in S$.

Nondeterministic choices over actions, probabilistic choices over states, and the race policy for exponentially distributed delays in the original MA are preserved in the corresponding $\mathbb{R}_{[0,1]}$-ULTRAS. This is exemplified in Fig. 4.

A notion of bisimilarity for probabilistic exponentially-timed processes was introduced in [7]. Below, we reformulate the definition in terms of the two distinct transition relations.

Definition 19. Let $(S, A, \longrightarrow, \leadsto)$ be an $M A$. An equivalence relation $\mathcal{B}$ over $S$ is a probabilistic exponentially-timed bisimulation iff, whenever $\left(s_{1}, s_{2}\right) \in \mathcal{B}$, then for all actions $a \in A$ and rates $\lambda \in \mathbb{R}_{>0}$ it holds that:

- For each $s_{1} \stackrel{a}{\longrightarrow} \mathcal{D}_{1}$ there exists $s_{2} \stackrel{a}{\longrightarrow} \mathcal{D}_{2}$ such that for all equivalence classes $C \in S / \mathcal{B}$ it holds that $\mathcal{D}_{1}(C)=\mathcal{D}_{2}(C)$.

- If $s_{1}$ has outgoing time-transitions, then $s_{2}$ has outgoing time-transitions too and for all equivalence classes $C \in S / \mathcal{B}$ it holds that:

$$
\sum_{s_{1} \stackrel{\lambda}{\sim} s_{1}^{\prime} \in C} \lambda=\sum_{s_{2} \stackrel{\lambda}{\sim} s_{2}^{\prime} \in C} \lambda
$$

We say that $s_{1}, s_{2} \in S$ are probabilistic exponentially-timed bisimilar, written $s_{1} \sim$ PEB $s_{2}$, iff there exists a probabilistic exponentially-timed bisimulation $\mathcal{B}$ over $S$ such that $\left(s_{1}, s_{2}\right) \in \mathcal{B}$.

The relation $\sim_{\mathrm{PEB}}$ over MA models coincides with the relation $\sim_{\mathrm{PTB}}$, dis over $\mathbb{R}_{[0,1]}$-ULTraS models given in Def. 13 . As a consequence, all the subsequent definitions and results in Sect. 5 also apply to $\mathbb{R}_{[0,1]}$-ULTrAS models corresponding to MA models.

Theorem 6. Let $(S, A, \longrightarrow, \sim)$ be an $M A$ and $\mathcal{U}=\left(S, A_{\mathcal{U}}, \longrightarrow \mathcal{U}\right)$ be the corresponding $\mathbb{R}_{[0,1]}$-ULTRAS. For all $s_{1}, s_{2} \in S$ :

$$
s_{1} \sim_{\mathrm{PEB}} s_{2} \Longleftrightarrow s_{1} \sim_{\mathrm{PTB}, \mathrm{dis}} s_{2}
$$




\section{Discussion and Conclusions}

In this paper, widely used timed models such as TA [1] (together with their underlying semantic model TLTS), PTA [12] (with their underlying PTLTS), and MA [7] have been put in a unifying view by encoding them in the ULTRAS framework [4] and by examining their bisimulation semantics [16, 20, 19, 7].

As immediate results of this work, we have been able to re-obtain the already existing timed bisimilarities and, most notably, to give new contributions. In particular, by naturally instantiating the ULTRAS general bisimilarity definition to the case of deterministically timed models - i.e., TLTS and TA - we have retrieved the same timed bisimilarity introduced in the literature (Thm. 1). Instead, when time is mixed with probability - i.e., for PTLTS, PTA, and MA models - we have found that the bisimilarities present in the literature, although expressible within the ULTRAS framework (Thms. 2 and 6), are different from the one that can be naturally obtained from ULTRAS. This has led us to introduce a new bisimilarity for those models (Def. 14 and Thm. 3), which we have called group-by-group and shown to be coarser than the original one (Thm. 4). Moreover, we have exhibited a modal logic characterization for the group-bygroup bisimilarity by using an interval-based variant of the logic in [14] (Thm. 5), while the original bisimilarity needs a much more expressive logic [19].

The ULTRAS-based encodings permit also more general considerations about the studied models. Firstly, the transition relation of the ULTRAS corresponding to a TA is functional and based on $\mathbb{B}$, whilst in the case of a PTA/MA it is not a function (because internal nondeterminism cannot be mixed with probabilities in the target state distributions of transitions) and it is necessarily based on $\mathbb{R}_{[0,1]}$. This stresses the higher expressivity of PTA/MA compared to TA with regard to describing state reachability. Furthermore, it evidences a structural analogy between PTA and MA that has not been addressed so far in the literature.

Secondly, the quantitative information related to time in TA/PTA/MA can be made disappear to a large extent, while quantitative information related to probabilities in PTA/MA cannot be abstracted. This underlines an important difference between time and probability. Time elapses independent of the occurrence of events and hence its passage can be viewed as an event in its own, which can thus be represented like the other events. Probabilities, instead, are inherently associated with the occurrence of events and must therefore be explicitly represented as event attributes.

Indeed, in our encodings time passing has been represented through special actions that encompass the duration/rate of delays. A purely qualitative representation of time based on a single special action $\epsilon$ is also possible and was used, for instance, in the construction of the region/zone graph and in the notion of time-abstract bisimilarity [15]. This supports a compact description of the state space of the ULTRAS corresponding to a TA/PTA, which is uncountable while this is not necessarily the case for an MA. The reason is the inherent difference between deterministic time, which needs the concrete representation of all possible delays, and exponentially distributed time, for which a symbolic representation based on rates is sufficient thanks to the memoryless property. 
A natural continuation of our work is to investigate trace and testing equivalences by applying their general definitions in [4] to the considered timed models.

\section{References}

1. R. Alur and D.L. Dill. A theory of timed automata. Theoretical Computer Science, 126:183-235, 1994.

2. B. Bérard, A. Petit, V. Diekert, and P. Gastin. Characterization of the expressive power of silent transitions in timed automata. Fundamenta Informaticae, 36:145$182,1998$.

3. M. Bernardo. On the expressiveness of Markovian process calculi with durational and durationless actions. In Proc. of GANDALF 2010, volume 25 of EPTCS, pages 199-213, 2010.

4. M. Bernardo, R. De Nicola, and M. Loreti. A uniform framework for modeling nondeterministic, probabilistic, stochastic, or mixed processes and their behavioral equivalences. Information and Computation, 225:29-82, 2013.

5. K. Cerans. Decidability of bisimulation equivalences for parallel timer processes. In Proc. of $C A V$ 1992, volume 663 of $L N C S$, pages 302-315. Springer, 1992.

6. F. Corradini. Absolute versus relative time in process algebras. Information and Computation, 156:122-172, 2000.

7. C. Eisentraut, H. Hermanns, and L. Zhang. On probabilistic automata in continuous time. In Proc. of LICS 2010, pages 342-351. IEEE-CS Press, 2010.

8. M. Hennessy and R. Milner. Algebraic laws for nondeterminism and concurrency. Journal of the ACM, 32:137-162, 1985.

9. T.A. Henzinger, X. Nicollin, J. Sifakis, and S. Yovine. Symbolic model checking for real-time systems. Information and Computation, 111:193-244, 1994.

10. H. Hermanns. Interactive Markov Chains. Springer, 2002. Volume 2428 of LNCS.

11. R.M. Keller. Formal verification of parallel programs. Communications of the ACM, 19:371-384, 1976.

12. M. Kwiatkowska, G. Norman, R. Segala, and J. Sproston. Automatic verification of real-time systems with discrete probability distributions. Theoretical Computer Science, 282:101-150, 2002.

13. R. Lanotte, A. Maggiolo-Schettini, and A. Troina. Weak bisimulation for probabilistic timed automata. Theoretical Computer Science, 411:4291-4322, 2010.

14. K.G. Larsen and A. Skou. Bisimulation through probabilistic testing. Information and Computation, 94:1-28, 1991.

15. K.G. Larsen and W. Yi. Time abstracted bisimulation: Implicit specifications and decidability. In Proc. of MFPS 1993, volume 802 of LNCS, pages 160-176. Springer, 1993.

16. F. Moller and C. Tofts. A temporal calculus of communicating systems. In Proc. of CONCUR 1990, volume 458 of LNCS, pages 401-415. Springer, 1990.

17. F. Moller and C. Tofts. Behavioural abstraction in TCCS. In Proc. of ICALP 1992, volume 623 of LNCS, pages 559-570. Springer, 1992.

18. R. Segala. Modeling and Verification of Randomized Distributed Real-Time Systems. PhD Thesis, 1995.

19. J. Sproston and A. Troina. Simulation and bisimulation for probabilistic timed automata. In Proc. of FORMATS 2010, volume 6246 of LNCS, pages 213-227. Springer, 2010.

20. W. Yi. CCS + time $=$ an interleaving model for real time systems. In Proc. of ICALP 1991, volume 510 of LNCS, pages 217-228. Springer, 1991. 


\section{Appendix: Proofs of Results}

Proof of Thm. 1. Let $s_{1}, s_{2} \in S$. Assume that $s_{1} \sim_{\mathrm{TB}} s_{2}$ due to some timed bisimulation $\mathcal{B}$ over $S$ such that $\left(s_{1}, s_{2}\right) \in \mathcal{B}$. This means that, whenever $\left(s_{1}^{\prime}, s_{2}^{\prime}\right) \in \mathcal{B}$, then for all $a \in A$ and $t \in \mathbb{R}_{\geq 0}$ it holds that:

- For each $s_{1}^{\prime} \stackrel{a}{\longrightarrow} s_{1}^{\prime \prime}\left(\right.$ resp. $\left.s_{2}^{\prime} \stackrel{a}{\longrightarrow} s_{2}^{\prime \prime}\right)$ there exists $s_{2}^{\prime} \stackrel{a}{\longrightarrow} s_{2}^{\prime \prime}\left(\right.$ resp. $\left.s_{1}^{\prime} \stackrel{a}{\longrightarrow} s_{1}^{\prime \prime}\right)$ such that $\left(s_{1}^{\prime \prime}, s_{2}^{\prime \prime}\right) \in \mathcal{B}$.

- For each $s_{1}^{\prime} \stackrel{t}{\sim} s_{1}^{\prime \prime}$ (resp. $s_{2}^{\prime} \stackrel{t}{\sim} s_{2}^{\prime \prime}$ ) there exists $s_{2}^{\prime} \stackrel{t}{\sim} s_{2}^{\prime \prime}$ (resp. $\left.s_{1}^{\prime} \stackrel{t}{\sim} s_{1}^{\prime \prime}\right)$ such that $\left(s_{1}^{\prime \prime}, s_{2}^{\prime \prime}\right) \in \mathcal{B}$.

Without loss of generality, we can suppose that $\mathcal{B}$ is an equivalence relation; should this not be the case, it suffices to take the reflexive, symmetric, and transitive closure of $\mathcal{B}$ as this is still a timed bisimulation. As a consequence, the assumption is equivalent to having that, whenever $\left(s_{1}^{\prime}, s_{2}^{\prime}\right) \in \mathcal{B}$, then for all $a \in A, t \in \mathbb{R}_{\geq 0}$, and $C \in S / \mathcal{B}$ it holds that:

- For each $s_{1}^{\prime} \stackrel{a}{\longrightarrow} s_{1}^{\prime \prime}$ such that $s_{1}^{\prime \prime} \in C$ there exists $s_{2}^{\prime} \stackrel{a}{\longrightarrow} s_{2}^{\prime \prime}$ such that $s_{2}^{\prime \prime} \in C$, and vice versa.

- For each $s_{1}^{\prime} \stackrel{t}{\rightarrow} s_{1}^{\prime \prime}$ such that $s_{1}^{\prime \prime} \in C$ there exists $s_{2}^{\prime} \stackrel{t}{\sim} s_{2}^{\prime \prime}$ such that $s_{2}^{\prime \prime} \in C$, and vice versa.

In turn, this is equivalent to having that, whenever $\left(s_{1}^{\prime}, s_{2}^{\prime}\right) \in \mathcal{B}$, then for all $a \in A, t \in \mathbb{R}_{\geq 0}$, and $C \in S / \mathcal{B}$ it holds that:

- There exists $s_{1}^{\prime \prime} \in C$ such that $s_{1}^{\prime} \stackrel{a}{\longrightarrow} s_{1}^{\prime \prime}$ iff there exists $s_{2}^{\prime \prime} \in C$ such that $s_{2}^{\prime} \stackrel{a}{\longrightarrow} s_{2}^{\prime \prime}$.

- There exists $s_{1}^{\prime \prime} \in C$ such that $s_{1}^{\prime} \stackrel{t}{\sim} s_{1}^{\prime \prime}$ iff there exists $s_{2}^{\prime \prime} \in C$ such that $s_{2}^{\prime} \stackrel{t}{\rightarrow} s_{2}^{\prime \prime}$.

Since for all $s \in S, a \in A, t \in \mathbb{R}_{\geq 0}$, and $\mathcal{G} \in 2^{S / \mathcal{B}}$ it holds that the existence of $s^{\prime} \in \bigcup \mathcal{G}$ such that $s \stackrel{a}{\longrightarrow} s^{\prime}$ (resp. $s \stackrel{t}{\sim} s^{\prime}$ ) corresponds to the existence of $s^{\prime} \in C$ such that $s \stackrel{a}{\longrightarrow} s^{\prime}$ (resp. $s \stackrel{t}{\sim} s^{\prime}$ ) for some $C \in \mathcal{G}$, we immediately derive that the assumption is equivalent to having that, whenever $\left(s_{1}^{\prime}, s_{2}^{\prime}\right) \in \mathcal{B}$, then for all $a \in A, t \in \mathbb{R}_{\geq 0}$, and $\mathcal{G} \in 2^{S / \mathcal{B}}$ :

- There exists $s_{1}^{\prime \prime} \in \bigcup \mathcal{G}$ such that $s_{1}^{\prime} \stackrel{a}{\longrightarrow} s_{1}^{\prime \prime}$ iff there exists $s_{2}^{\prime \prime} \in \bigcup \mathcal{G}$ such that $s_{2}^{\prime} \stackrel{a}{\longrightarrow} s_{2}^{\prime \prime}$.

- There exists $s_{1}^{\prime \prime} \in \bigcup \mathcal{G}$ such that $s_{1}^{\prime} \stackrel{t}{\sim} s_{1}^{\prime \prime}$ iff there exists $s_{2}^{\prime \prime} \in \bigcup \mathcal{G}$ such that $s_{2}^{\prime} \stackrel{t}{\rightarrow} s_{2}^{\prime \prime}$.

Since for all $s \in S, a \in A_{\mathcal{U}}$, and $\mathcal{G} \in 2^{S / \mathcal{B}}$ it holds that:

$$
\mathcal{M}_{\mathbb{B}}(s, a, \cup \mathcal{G})=\bigvee_{s^{\prime} \in \cup \mathcal{G}} \mathcal{D}_{s, a}\left(s^{\prime}\right)= \begin{cases}\left(\exists s^{\prime} \in \bigcup \mathcal{G} . s \stackrel{a}{\longrightarrow} s^{\prime}\right) & \text { if } a \in A \\ \left(\exists s^{\prime} \in \bigcup \mathcal{G} . s \stackrel{t}{\sim} s^{\prime}\right) & \text { if } a=\epsilon(t)\end{cases}
$$

we further derive that the assumption is equivalent to having that, whenever 
$\left(s_{1}^{\prime}, s_{2}^{\prime}\right) \in \mathcal{B}$, then for all $a \in A_{\mathcal{U}}$ and $\mathcal{G} \in 2^{S / \mathcal{B}}$ it holds that:

$$
\mathcal{M}_{\mathbb{B}}\left(s_{1}^{\prime}, a, \bigcup \mathcal{G}\right)=\mathcal{M}_{\mathbb{B}}\left(s_{2}^{\prime}, a, \bigcup \mathcal{G}\right)
$$

This means that $\mathcal{B}$ is an $\mathcal{M}_{\mathbb{B}}$-bisimulation such that $\left(s_{1}, s_{2}\right) \in \mathcal{B}$. In other words, $s_{1} \sim \sim_{\mathrm{B}, \mathcal{M}_{\mathbb{B}}} s_{2}$.

Proof of Thm. 2. It follows immediately from the translation in Def. 11 after observing that the discriminating power of $\sim_{\text {PTB }}$ does not change if, in the definition of the equivalence (Def. 12), we replace equivalence classes with groups of equivalence classes.

Proof of Thm. 3. Let $s_{1}, s_{2} \in S$. Assume that $s_{1} \sim_{\mathrm{PTB}, \mathrm{gbg}} s_{2}$ due to some probabilistic timed group-by-group bisimulation $\mathcal{B}$ over $S$ such that $\left(s_{1}, s_{2}\right) \in \mathcal{B}$. This means that, whenever $\left(s_{1}^{\prime}, s_{2}^{\prime}\right) \in \mathcal{B}$, then for all $a \in A$ and $\mathcal{G} \in 2^{S / \mathcal{B}}$ it holds that:

- For each $s_{1}^{\prime} \stackrel{a}{\longrightarrow} \mathcal{D}_{1}$ there exists $s_{2}^{\prime} \stackrel{a}{\longrightarrow} \mathcal{D}_{2}$ such that $\mathcal{D}_{1}(\bigcup \mathcal{G})=\mathcal{D}_{2}(\bigcup \mathcal{G})$.

In other words, whenever $\left(s_{1}^{\prime}, s_{2}^{\prime}\right) \in \mathcal{B}$, then for all $a \in A$ and $\mathcal{G} \in 2^{S / \mathcal{B}}$ :

or equivalently:

$$
\begin{aligned}
\underbrace{\prime}_{1} \stackrel{a}{\longrightarrow} \mathcal{D}_{1} & \left\{\mathcal{D}_{1}(\bigcup \mathcal{G})\right\} \subseteq \underset{s_{2}^{\prime} \stackrel{a}{\longrightarrow} \mathcal{D}_{2}}{\bigcup}\left\{\mathcal{D}_{2}(\bigcup \mathcal{G})\right\} \\
\operatorname{s}_{2}^{\prime} \stackrel{a}{\longrightarrow} \mathcal{D}_{2} & \left\{\mathcal{D}_{2}(\bigcup \mathcal{G})\right\} \subseteq \bigcup_{s_{1}^{\prime} \stackrel{a}{\longrightarrow} \mathcal{D}_{1}}^{\bigcup}\left\{\mathcal{D}_{1}(\bigcup \mathcal{G})\right\}
\end{aligned}
$$

$$
\bigcup_{s_{1}^{\prime} \stackrel{a}{\longrightarrow} \mathcal{D}_{1}}\left\{\mathcal{D}_{1}(\bigcup \mathcal{G})\right\}=\bigcup_{s_{2}^{\prime} \stackrel{a}{\longrightarrow} \mathcal{D}_{2}}\left\{\mathcal{D}_{2}(\bigcup \mathcal{G})\right\}
$$

Since for all $s \in S, a \in A$, and $\mathcal{G} \in 2^{S / \mathcal{B}}$ it holds that $\mathcal{M}_{2^{\mathbb{R}}[0,1]}(s, a, \bigcup \mathcal{G})=\{0\}$ when $s$ has no $a$-transition, otherwise:

$$
\mathcal{M}_{2:[0,1]}^{\mathbb{R}}(s, a, \bigcup \mathcal{G})=\bigcup_{s \stackrel{a}{\longrightarrow} \mathcal{D}}\left\{\sum_{s^{\prime} \in \bigcup \mathcal{G}} \mathcal{D}\left(s^{\prime}\right)\right\}=\bigcup_{s \stackrel{a}{\longrightarrow} \mathcal{D}}\{\mathcal{D}(\bigcup \mathcal{G})\}
$$

we derive that the assumption is equivalent to having that, whenever $\left(s_{1}^{\prime}, s_{2}^{\prime}\right) \in \mathcal{B}$, then for all $a \in A$ and $\mathcal{G} \in 2^{S / \mathcal{B}}$ :

$$
\mathcal{M}_{2 \cdot[0,1]}\left(s_{1}^{\prime}, a, \bigcup \mathcal{G}\right)=\mathcal{M}_{2 \mathbb{R}_{[0,1]}}\left(s_{2}^{\prime}, a, \bigcup \mathcal{G}\right)
$$

This means that $\mathcal{B}$ is an $\mathcal{M}_{2^{\mathbb{R}}[0,1]}$-bisimulation such that $\left(s_{1}, s_{2}\right) \in \mathcal{B}$. In other words, $s_{1} \sim_{\mathrm{B}, \mathcal{M}_{2} \mathbb{R}_{[0,1]}} s_{2}$.

Proof of Thm. 4. A straightforward consequence of the fact that a probabilistic timed group-distribution bisimulation is also a probabilistic timed group-bygroup bisimulation, as can be easily seen by taking the same fully matching transitions considered in the group-distribution bisimulation.

Proof of Thm. 5. The result holds when $\mathcal{U}$ is image finite and satisfies the minimal probability assumption. $\mathcal{U}$ is image finite [8] iff for all $s \in S$ and $a \in A_{\mathcal{U}}$ the set $\left\{\mathcal{D} \in \operatorname{Distr}_{\mathrm{cs}}(S) \mid s \stackrel{a}{\longrightarrow} \mathcal{U} \mathcal{D}\right\}$ is finite. $\mathcal{U}$ satisfies the minimal probability assumption [14] iff there exists $\epsilon \in \mathbb{R}_{>0}$ such that, whenever $s \stackrel{a}{\longrightarrow} \mathcal{U} \mathcal{D}$, then $\mathcal{D}\left(s^{\prime}\right) \geq \epsilon$ for all $s^{\prime} \in \operatorname{supp}(\mathcal{D})$; this implies that $\operatorname{supp}(\mathcal{D})$ is finite because it can have at most $\lceil 1 / \epsilon\rceil$ elements.

The proof of the result is divided into two parts. In the first part, we provide an alternative characterization of $\sim_{\mathrm{PTB}, \mathrm{gbg}}$ as the limit of a sequence of equivalence relations $\sim_{\mathrm{PTB}, \mathrm{gbg}}^{i}, i \in \mathbb{N}$, which are inductively defined as follows: 
$-\sim_{\mathrm{PTB}, \mathrm{gbg}}^{0}=S \times S$.

$-\sim_{\mathrm{PTB}, \mathrm{gbg}}^{i+1}$ is the set of all pairs $\left(s_{1}, s_{2}\right) \in \sim_{\mathrm{PTB}, \mathrm{gbg}}^{i}$ such that for all actions $a \in A_{\mathcal{U}}$ and groups of equivalence classes $\mathcal{G} \in 2^{S / \sim_{\mathrm{PTB}, \mathrm{gbg}}^{i}}$ it holds that for each $s_{1} \stackrel{a}{\longrightarrow} \mathcal{U} \mathcal{D}_{1}$ there exists $s_{2} \stackrel{a}{\longrightarrow} \mathcal{U} \mathcal{D}_{2}$ such that $\mathcal{D}_{1}(\cup \mathcal{G})=\mathcal{D}_{2}(\cup \mathcal{G})$.

Denoting by $\sim^{\prime}$ the relation $\bigcap_{i \in \mathbb{N}} \sim_{\mathrm{PTB}, \mathrm{gbg}}^{i}$, we prove that $\sim^{\prime}=\sim_{\mathrm{PTB}, \mathrm{gbg}}$. Firstly, we observe what follows:

$-\sim^{\prime}$ is an equivalence relation because so is $\sim_{\mathrm{PTB}, \mathrm{gbg}}^{i}$ for all $i \in \mathbb{N}$.

- Given $C \in S / \sim^{\prime}$ and $i \in \mathbb{N}$, there exists a unique element $C_{i}$ in $S / \sim_{\mathrm{PTB}, \mathrm{gbg}}^{i}$ such that $C_{i} \supseteq C$, and hence $C=\bigcap_{i \in \mathbb{N}} C_{i}$ with $C_{i_{1}} \supseteq C_{i_{2}}$ for $i_{1} \leq i_{2}$.

- As a consequence, given $\mathcal{G} \in 2^{S / \sim^{\prime}}$ and $i \in \mathbb{N}$, there exists a unique element $\mathcal{G}_{i}$ in $2^{S / \sim_{\mathrm{PTB}, \mathrm{gbg}}^{i}}$ such that every class in $\mathcal{G}_{i}$ contains some class in $\mathcal{G}$, and hence $\bigcup \mathcal{G}=\bigcap_{i \in \mathbb{N}}\left(\bigcup \mathcal{G}_{i}\right)$ with $\bigcup \mathcal{G}_{i_{1}} \supseteq \bigcup \mathcal{G}_{i_{2}}$ for $i_{1} \leq i_{2}$.

- Moreover, if $s \stackrel{a}{\longrightarrow} \mathcal{U} \mathcal{D}$, then $\mathcal{D}(\bigcup \mathcal{G})=\inf _{i \in \mathbb{N}} \mathcal{D}\left(\bigcup \mathcal{G}_{i}\right)$. In fact, observing that for all $i \in \mathbb{N}$ it holds that $\mathcal{D}\left(\bigcup \mathcal{G}_{i}\right) \geq \mathcal{D}(\bigcup \mathcal{G})$ because $\bigcup \mathcal{G}_{i} \supseteq \bigcup \mathcal{G}$, if we let $p=\inf _{i \in \mathbb{N}} \mathcal{D}\left(\bigcup \mathcal{G}_{i}\right)$, then $p \geq \mathcal{D}(\bigcup \mathcal{G})$ because $\mathcal{D}(\bigcup \mathcal{G})$ is a lower bound of the sequence $\left(\mathcal{D}\left(\bigcup \mathcal{G}_{i}\right)\right)_{i \in \mathbb{N}}$ and $p$ is the greatest lower bound of that sequence. Suppose $p>\mathcal{D}(\bigcup \mathcal{G})$ and let $\delta=p-\mathcal{D}(\bigcup \mathcal{G})$. Since $\delta>0$ and the summation $\mathcal{D}(S \backslash \cup \mathcal{G})$ satisfies $\sum_{s \in S \backslash \cup \mathcal{G}} \mathcal{D}(s) \leq 1$ and hence converges, there exists a finite subset $X$ of $S \backslash \bigcup \mathcal{G}$ such that the rest $\mathcal{D}((S \backslash \cup \mathcal{G}) \backslash X)$ of the previously considered summation satisfies $\sum_{s \in(S \backslash \cup \mathcal{G}) \backslash X} \mathcal{D}(s)<\delta$. Let $Y=(S \backslash \bigcup \mathcal{G}) \backslash X$. For all $i \in \mathbb{N}$, it holds that:

$$
\bigcup \mathcal{G}_{i}=\bigcup \mathcal{G} \cup\left(Y \cap \bigcup \mathcal{G}_{i}\right) \cup\left(X \cap \bigcup \mathcal{G}_{i}\right)
$$

where the three sets on the right-hand side are pairwise disjoint and hence:

$$
\begin{aligned}
\mathcal{D}\left(\bigcup \mathcal{G}_{i}\right) & =\mathcal{D}(\bigcup \mathcal{G})+\mathcal{D}\left(Y \cap \bigcup \mathcal{G}_{i}\right)+\mathcal{D}\left(X \cap \bigcup \mathcal{G}_{i}\right) \\
& \leq \mathcal{D}(\bigcup \mathcal{G})+\mathcal{D}(Y)+\mathcal{D}\left(X \cap \bigcup \mathcal{G}_{i}\right) \\
& <\mathcal{D}(\bigcup \mathcal{G})+\delta+\mathcal{D}\left(X \cap \bigcup \mathcal{G}_{i}\right) \\
& =p+\mathcal{D}\left(X \cap \bigcup \mathcal{G}_{i}\right)
\end{aligned}
$$

From the inequality above and $\mathcal{D}\left(\bigcup \mathcal{G}_{i}\right) \geq p$, we derive that:

$$
\mathcal{D}\left(X \cap \bigcup \mathcal{G}_{i}\right)>\mathcal{D}\left(\bigcup \mathcal{G}_{i}\right)-p \geq 0
$$

and hence $X \cap \bigcup \mathcal{G}_{i} \neq \emptyset$ for all $i \in \mathbb{N}$. As a consequence, $X \cap \bigcup \mathcal{G} \neq \emptyset$ because $X$ is finite and $\bigcup \mathcal{G}_{0} \supseteq \bigcup \mathcal{G}_{1} \supseteq \ldots$. This contradicts the fact that $X$ is a subset of $S \backslash \bigcup \mathcal{G}$. Therefore, it must be $p=\mathcal{D}(\bigcup \mathcal{G})$.

Secondly, it holds that $\sim^{\prime} \supseteq \sim_{\text {PTB,gbg }}$ because $\sim_{\text {PTB,gbg }}^{i} \supseteq \sim_{\text {PTB,gbg }}$ for all $i \in \mathbb{N}$ as we now show by proceeding by induction on $i$ :

- If $i=0$, then $\sim_{\mathrm{PTB}, \mathrm{gbg}}^{i}=S \times S \supseteq \sim_{\mathrm{PTB}, \mathrm{gbg}}$.

- Let $i$ be an element of $\mathbb{N}$ for which the result holds and consider $i+1$. If $s_{1}, s_{2} \in S$ satisfy $s_{1} \sim_{\mathrm{PTB}, \mathrm{gbg}} s_{2}$, then:

- For all $a \in A_{\mathcal{U}}$ and $\mathcal{G} \in 2^{S / \sim_{\mathrm{PTB}, \mathrm{gbg}}}$, it holds that for each $s_{1} \stackrel{a}{\longrightarrow} \mathcal{U} \mathcal{D}_{1}$ there exists $s_{2} \stackrel{a}{\longrightarrow} \mathcal{U} \mathcal{D}_{2}$ such that $\mathcal{D}_{1}(\bigcup \mathcal{G})=\mathcal{D}_{2}(\bigcup \mathcal{G})$.

- $s_{1} \sim_{\mathrm{PTB}, \mathrm{gbg}}^{i} s_{2}$ because $\sim_{\mathrm{PTB}, \mathrm{gbg}}^{i} \supseteq \sim_{\mathrm{PTB}, \mathrm{gbg}}$ by the induction hypothesis. 
Since every equivalence class of $\sim_{\mathrm{PTB}, \mathrm{gbg}}^{i}$ is equal to the union of some equivalence classes of $\sim_{\mathrm{PTB}, \text { gbg }}$ and hence the union of equivalence classes in every group $\mathcal{G}^{\prime}$ of $\sim_{\mathrm{PTB}, \mathrm{gbg}}^{i}$ is equal to the union of the equivalence classes in some group $\mathcal{G}$ of $\sim_{\text {PTB,gbg }}$, we derive that for all $a \in A_{\mathcal{U}}$ and $\mathcal{G}^{\prime} \in 2^{S / \sim_{\text {PTB,gbg }}^{i}}$ it holds that for each $s_{1} \stackrel{a}{\longrightarrow} \mathcal{U} \mathcal{D}_{1}$ there exists $s_{2} \stackrel{a}{\longrightarrow} \mathcal{U} \mathcal{D}_{2}$ such that:

This means that $s_{1} \sim_{\mathrm{PTB}, \mathrm{gbg}}^{i+1} s_{2}$.

$$
\mathcal{D}_{1}\left(\bigcup \mathcal{G}^{\prime}\right)=\mathcal{D}_{1}(\bigcup \mathcal{G})=\mathcal{D}_{2}(\bigcup \mathcal{G})=\mathcal{D}_{2}\left(\bigcup \mathcal{G}^{\prime}\right)
$$

Thirdly, we prove that $\sim^{\prime} \subseteq \sim_{\mathrm{PTB}}$,gbg by showing that $\sim^{\prime}$ is a probabilistic timed group-by-group bisimulation. Suppose that $s_{1}, s_{2} \in S$ satisfy $s_{1} \sim^{\prime} s_{2}$ and, given $a \in A_{\mathcal{U}}$ and $\mathcal{G} \in 2^{S / \sim^{\prime}}$, assume that $s_{1} \stackrel{a}{\longrightarrow} \mathcal{U} \mathcal{D}_{1}$. Then $\mathcal{D}_{1}(\bigcup \mathcal{G})=\inf _{i \in \mathbb{N}} \mathcal{D}_{1}\left(\bigcup \mathcal{G}_{i}\right)$ where each $\mathcal{G}_{i}$ is the unique element in $2^{S / \sim_{\mathrm{PTB}, \mathrm{gbg}}^{i}}$ such that every class in $\mathcal{G}_{i}$ contains some class in $\mathcal{G}$.

Observing that $\sim_{\text {PTB,gbg }}^{0}$ induces a single equivalence class equal to $S$ and hence $\mathcal{D}_{1}\left(\bigcup \mathcal{G}_{0}\right)=\mathcal{D}_{1}(S)=1$, from $s_{1} \sim^{\prime} s_{2}$ and $s_{1} \stackrel{a}{\longrightarrow} \mathcal{U} \mathcal{D}_{1}$ it follows that for all $i \in \mathbb{N}_{\geq 1}$ there exists $s_{2} \stackrel{a}{\longrightarrow} \mathcal{U} \mathcal{D}_{2, i}$ such that $\mathcal{D}_{1}\left(\bigcup \mathcal{G}_{i-1}\right)=\mathcal{D}_{2, i}\left(\bigcup \mathcal{G}_{i-1}\right)$. Since $\mathcal{U}$ is image finite, the set $\left\{\mathcal{D}_{2, i} \mid i \in \mathbb{N}_{\geq 1}\right\}$ is finite and we enumerate it as $\left\{D_{2}^{1}, \ldots, D_{2}^{k}\right\}$. For each $j \in\{1, \ldots, k\}$, we also let $I_{j}$ be the set of indexes $i \in \mathbb{N}_{\geq 1}$ such that $D_{1}\left(\bigcup \mathcal{G}_{i-1}\right)=D_{2}^{j}\left(\bigcup \mathcal{G}_{i-1}\right)$. At least one set in $\left\{I_{1}, \ldots, I_{k}\right\}$ is infinite. Indeed, if every $I_{j}$ were finite, then there would exist an integer $i$ such that $i \notin I_{j}$ for each $j \in\{1, \ldots, k\}$. Hence, there would exists a group $\mathcal{G}_{i-1}$ such that $D_{1}\left(\bigcup \mathcal{G}_{i-1}\right) \neq D_{2}^{j}\left(\bigcup \mathcal{G}_{i-1}\right)$ for each $j \in\{1, \ldots, k\}$. However, this implies $s_{1} \chi_{\mathrm{PTB}, \mathrm{gbg}}^{i} s_{2}$, which whould contradict the assumption $s_{1} \sim^{\prime} s_{2}$.

Let $j^{\prime}$ be such that $I_{j^{\prime}}$ is infinite. We have that:

$$
\begin{aligned}
D_{1}(\mathcal{G}) & =\inf _{i \in \mathbb{N}} \mathcal{D}_{1}\left(\bigcup \mathcal{G}_{i}\right) \\
& =\inf _{i \in I_{j^{\prime}}} \mathcal{D}_{1}\left(\bigcup \mathcal{G}_{i}\right) \\
& =\inf _{i \in I_{j^{\prime}}} \mathcal{D}_{2}^{j^{\prime}}\left(\bigcup \mathcal{G}_{i}\right) \\
& =\inf _{i \in \mathbb{N}} \mathcal{D}_{2}^{j^{\prime}}\left(\bigcup \mathcal{G}_{i}\right)=D_{2}^{j^{\prime}}(\mathcal{G})
\end{aligned}
$$

where the equalities (1) and (2) above derive from the fact that $\left(D_{1}\left(\mathcal{G}_{i}\right)\right)_{i \in I_{j}^{\prime}}$ and $\left(D_{2}^{j^{\prime}}\left(\mathcal{G}_{i}\right)\right)_{i \in I_{j}^{\prime}}$ are infinite subsequences of $\left(D_{1}\left(\mathcal{G}_{i}\right)\right)_{i \in \mathbb{N}}$ and $\left(D_{2}^{j^{\prime}}\left(\mathcal{G}_{i}\right)\right)_{i \in \mathbb{N}}$, respectively, and therefore they have the same infimum. In conclusion, we have that $\sim^{\prime}$ is a probabilistic timed group-by-group bisimulation.

In the second part of the proof, we show that $s_{1}, s_{2} \in S$ are equated by $\sim_{\mathrm{PTB}, \mathrm{gbg}}^{i}$ iff they satisfy the same formulae in $\mathbb{F}_{\text {IPML }}^{i}$, which is the set of formulae in IPML whose maximum number of nested diamond operators is at most $i$. Denoting by $\mathcal{F}_{\text {IPML }}^{i}(s)$ the set of formulae in $\mathbb{F}_{\text {IPML }}^{i}$ satisfied by $s \in S$, we proceed by induction on $i \in \mathbb{N}$.

Let $i=0$. Since $\sim_{\text {PTB,gbg }}^{0}=S \times S$ and $\mathcal{F}_{\text {IPML }}^{0}(s)=\left\{\phi \in \mathbb{F}_{\text {IPML }}^{0} \mid \phi \equiv\right.$ true $\}$ for all $s \in S$, it trivially holds that:

$$
s_{1} \sim_{\text {PTB,gbg }}^{0} s_{2} \Longleftrightarrow \mathcal{F}_{\text {IPML }}^{0}\left(s_{1}\right)=\mathcal{F}_{\text {IPML }}^{0}\left(s_{2}\right)
$$

Let $i \in \mathbb{N}$ and suppose that for all $j=0, \ldots, i$ it holds that:

$$
s_{1} \sim_{\text {PTB }, \text { gbg }}^{j} s_{2} \Longleftrightarrow \mathcal{F}_{\text {IPML }}^{j}\left(s_{1}\right)=\mathcal{F}_{\text {IPML }}^{j}\left(s_{2}\right)
$$

We prove that both implications hold also for $i+1$ by reasoning on their corresponding contrapositive statements, i.e., we prove that: 


$$
\mathcal{F}_{\text {IPML }}^{i+1}\left(s_{1}\right) \neq \mathcal{F}_{\text {IPML }}^{i+1}\left(s_{2}\right) \Longleftrightarrow s_{1} \chi_{\text {PTB }, \text { gbg }}^{i+1} s_{2}
$$

$(\Longrightarrow)$ If $\mathcal{F}_{\text {IPML }}^{i+1}\left(s_{1}\right) \neq \mathcal{F}_{\text {IPML }}^{i+1}\left(s_{2}\right)$, then there are two cases:

- If $\mathcal{F}_{\text {IPML }}^{i}\left(s_{1}\right) \neq \mathcal{F}_{\text {IPML }}^{i}\left(s_{2}\right)$, then by the induction hypothesis it holds that $s_{1} \mathcal{L}_{\mathrm{PTB}, \mathrm{gbg}}^{i} s_{2}$ and hence $s_{1} \mathcal{\chi}_{\mathrm{PTB}, \mathrm{gbg}}^{i+1} s_{2}$.

- If $\mathcal{F}_{\text {IPML }}^{i}\left(s_{1}\right)=\mathcal{F}_{\text {IPML }}^{i}\left(s_{2}\right)$, then from $\mathcal{F}_{\text {IPML }}^{i+1}\left(s_{1}\right) \neq \mathcal{F}_{\text {IPML }}^{i+1}\left(s_{2}\right)$ it follows that there exists $\phi \in \mathbb{F}_{\text {IPML }}^{i+1}$ such that $s_{1} \in \mathcal{M} \llbracket \phi \rrbracket$ and $s_{2} \notin \mathcal{M} \llbracket \phi \rrbracket$. We now proceed by induction on the syntactical structure of $\phi$. Here we only consider the case $\phi=\langle a\rangle_{\left[p_{1}, p_{2}\right]} \phi^{\prime}$ because the other cases are routine.

From $s_{1} \in \mathcal{M} \llbracket\langle a\rangle_{\left[p_{1}, p_{2}\right]} \phi^{\prime} \rrbracket$ and $s_{2} \notin \mathcal{M} \llbracket\langle a\rangle_{\left[p_{1}, p_{2}\right]} \phi^{\prime} \rrbracket$, it follows that:

- $p_{1} \leq \mathcal{D}_{1}\left(\mathcal{M} \llbracket \phi^{\prime} \rrbracket\right) \leq p_{2}$ for some $\mathcal{D}_{1}$ such that $s_{1} \stackrel{a}{\longrightarrow} \mathcal{U} \mathcal{D}_{1}$.

- $\mathcal{D}_{2}\left(\mathcal{M} \llbracket \phi^{\prime} \rrbracket\right)<p_{1}$ or $\mathcal{D}_{2}\left(\mathcal{M} \llbracket \phi^{\prime} \rrbracket\right)>p_{2}$ for all $\mathcal{D}_{2}$ such that $s_{2} \stackrel{a}{\longrightarrow} \mathcal{U} \mathcal{D}_{2}$.

Since $\phi^{\prime} \in \mathbb{F}_{\text {IPML }}^{i}$, by the induction hypothesis there exists $\mathcal{G} \in 2^{S / \sim_{\mathrm{PTB}, \mathrm{gbg}}^{i}}$ such that $\bigcup_{C \in \mathcal{G}} C=\mathcal{M} \llbracket \phi^{\prime} \rrbracket$. Then:

- $\mathcal{D}_{1}(\bigcup \mathcal{G})=q \in \mathbb{R}_{\left[p_{1}, p_{2}\right]}$.

- $\mathcal{D}_{2}(\bigcup \mathcal{G}) \neq q$ for all $\mathcal{D}_{2}$ such that $s_{2} \stackrel{a}{\longrightarrow} \mathcal{U} \mathcal{D}_{2}$.

Therefore $s_{1} \mathcal{\chi}_{\mathrm{PTB}, \mathrm{gbg}}^{i+1} s_{2}$.

$(\Longleftarrow)$ If $s_{1} \mathcal{\psi}_{\mathrm{PTB}, \mathrm{gbg}}^{i+1} s_{2}$, then there are two cases:

- If $s_{1} \not \chi_{\text {PTB,gbg }}^{i} s_{2}$, then by the induction hypothesis it holds that $\mathcal{F}_{\text {IPML }}^{i}\left(s_{1}\right) \neq$ $\mathcal{F}_{\text {IPML }}^{i}\left(s_{2}\right)$ and hence $\mathcal{F}_{\text {IPML }}^{i+1}\left(s_{1}\right) \neq \mathcal{F}_{\text {IPML }}^{i+1}\left(s_{2}\right)$.

- If $s_{1} \sim_{\mathrm{PTB}, \mathrm{gbg}}^{i} s_{2}$, then from $s_{1} \mathcal{\chi}_{\mathrm{PTB}, \mathrm{gbg}}^{i+1} s_{2}$ it follows that there exist $p \in \mathbb{R}_{[0,1]}$ and $\mathcal{G} \in 2^{S / \sim_{\mathrm{PTB}, \mathrm{gbg}}^{i}}$ such that:

- $\mathcal{D}_{1}(\bigcup \mathcal{G})=p$ for some $\mathcal{D}_{1}$ such that $s_{1} \stackrel{a}{\longrightarrow} \mathcal{U} \mathcal{D}_{1}$.

- $\mathcal{D}_{2}(\bigcup \mathcal{G}) \neq p$ for all $\mathcal{D}_{2}$ such that $s_{2} \stackrel{a}{\longrightarrow} \mathcal{U} \mathcal{D}_{2}$.

Let $\mathcal{G}_{1}=\left\{C \in S / \sim_{\mathrm{PTB}, \mathrm{gbg}}^{i} \mid \mathcal{D}_{1}(C)>0\right\}$ and $\mathcal{G}_{2}=\left\{C \in S / \sim_{\mathrm{PTB}, \mathrm{gbg}}^{i} \mid\right.$ $\left.\exists \mathcal{D}_{2} . s_{2} \stackrel{a}{\longrightarrow} \mathcal{U} \mathcal{D}_{2} \wedge \mathcal{D}_{2}(C)>0\right\}$. Thanks to the assumptions of image finiteness and minimal probability, both $\mathcal{G}_{1}$ and $\mathcal{G}_{2}$ are finite.

By the induction hypothesis, there exists a distinguishing formula $\phi_{<C_{1}, C_{2}>} \in$ $\mathbb{F}_{\mathrm{IPML}}^{i}$ for all $C_{1}$ and $C_{2}$ in $S / \sim_{\mathrm{PTB}, \mathrm{gbg}}^{i}$ such that $C_{1} \neq C_{2}$, i.e.:

Then:

$$
\begin{aligned}
& C_{1} \subseteq \mathcal{M} \llbracket \phi_{<C_{1}, C_{2}>} \rrbracket \\
& C_{2} \cap \mathcal{M} \llbracket \phi_{<C_{1}, C_{2}>} \rrbracket=\emptyset
\end{aligned}
$$

$$
\phi_{\mathcal{G}}=\bigvee_{C \in \mathcal{G}}\left(\bigwedge_{C_{1} \in \mathcal{G}_{1} \backslash\{C\}} \phi_{<C, C_{1}>} \wedge \bigwedge_{C_{2} \in \mathcal{G}_{2} \backslash\{C\}} \phi_{<C, C_{2}>}\right)
$$

where $\bigvee_{i \in I} \phi_{i}=\neg \bigwedge_{i \in I} \neg \phi_{i}$ for $I$ finite and $\bigwedge_{i \in I} \phi_{i}=$ true for $I=\emptyset$, yields a distinguishing formula for $s_{1}$ and $s_{2}$ because:

- $s_{1} \in \mathcal{M} \llbracket\langle a\rangle_{[p, p]} \phi_{\mathcal{G}} \rrbracket$.

- $s_{2} \notin \mathcal{M} \llbracket\langle a\rangle_{[p, p]} \phi_{\mathcal{G}} \rrbracket$.

Since $\langle a\rangle_{[p, p]} \phi_{\mathcal{G}} \in \mathbb{F}_{\text {IPML }}^{i+1}$, we derive that $\mathcal{F}_{\text {IPML }}^{i+1}\left(s_{1}\right) \neq \mathcal{F}_{\text {IPML }}^{i+1}\left(s_{2}\right)$. 
Proof of Thm. 6. It follows immediately from the translation in Def. 18 after observing that the discriminating power of $\sim_{\mathrm{PEB}}$ does not change if, in the definition of the equivalence (Def. 19), we replace equivalence classes with groups of equivalence classes.

In particular, note that, if $s_{1} \sim_{\mathrm{PEB}} s_{2}$ and both states have outgoing timetransitions, then in the corresponding $\mathbb{R}_{[0,1]}$-ULTRAS each of the two states has a single outgoing transition representing time passing. The label of this transition contains the same rate for both states as can be seen by taking $\mathcal{G}$ equal to the set of all equivalence classes - so that $\bigcup \mathcal{G}=S$ - in the second clause of the group-based version of Def. 19. 